Original

\title{
Changes in Load Pressure During Walking in Kyphotic Patients
}

\author{
Eiji Shiotani, Yutaka Hiraizumi and Etsuo Fujimaki
}

\begin{abstract}
Dynamic changes during walking were evaluated in kyphotic patients by measuring load pressure using an F-SCAN, a sensor that facilitates measurement of plantar pressure. The subjects consisted of kyphotic patients demonstrating severe thoracic kyphosis and more than 1 old compression fracture at the transitional zone between the thoracic and lumbar vertebrae. The results of analysis in these patients were compared with those in healthy subjects. As a structural evaluation, plumb-line shift from the central sacral line (P-CSL) in a standing profile was correlated with changes in load pressure. The types of central loci of the plantar pressure were analyzed in these two groups, the total load between heel contact and toe off was evaluated in the respective locus, and the integrates and load duration were also determined in the respective compartments after dividing the plantar region into the following 4 compartments: heel, plantar arch, anterior foot, and toe. kyphotic patients maintain their anteroposterior equilibrium during walking by shifting the centroid in the posterior direction, a P-CSL. Therefore, the F-SCAN demonstrated an increased proportion of weightbearing at the heel. Points of application of the centroid in the planta pedis were almost identical between kyphotic patients and healthy subjects during natural standing. Therefore, it was considered that kyphotic patients maintain their anteroposterior equilibrium by increased integrates at the heel (prolongation of load times at the heel). That is, the interval between heel contact and plantar contact tended to increase with the degree of anterior P-CSL.
\end{abstract}

Key words : kyphosis, loaded pressure, F-SCAN

\section{Introduction}

In general, during the stance phase of locomotion, the centroid of the body is not identical to the axis of the grounded leg. Therefore, weight-bearing joints in the lower extremities need to be coordinated with the spinal column to maintain balanced standing. However, spinal deformity such as kyphosis impairs coordination between weight-bearing joints in the lower extremities with the spinal column, resulting in unstable walking and difficulties in daily living. In the present study we examined load pressure during walking in kyphotic patients. An F-SCAN was used to determine whether the shifting of the centroid caused by structural changes in the spinal column is reflected in load pressure.

Department of Orthopaedic Surgery, Showa University School of Medicine 1-5-8 Hatanodai, Shinagawa-ku, Tokyo 142-8666, Japan 


\section{Subjects and Methods}

\section{Subjects}

Between April 1994 and April 1999, 36 patients (17 males and 19 females) with kyphosis without lower back pain and or pain in weight-bearing joints (hip, knee, and ankle) during walking were treated in our hospital. Lateral roentgenograms of these patients demonstrated severe thoracic kyphosis and more than 1 compression fracture at the transitional zone between the thoracic and lumbar vertebrae. The present study evaluated these 36 kyphotic patients. All patients could walk without a cane, and had balanced stable walking ability. The age of the patients ranged from 58 to 80 years (mean: 71.1 years). The controls consisted of 20 healthy subjects with stable walking ability ( 8 males and 12 females) aged between 22 and 61 years of age (mean : 64.5 years). The height of control subjects ranged from 146 to $171 \mathrm{~cm}$ (mean : $158.0 \mathrm{~cm}$ ), while that of kyphotic patients ranged from 141 to $161 \mathrm{~cm}$ (mean : $151.1 \mathrm{~cm}$ ). There were no significant differences in age or height between the two groups.

\section{Methods}

\section{Measurment of load pressure}

We chose the F-SCAN from various tactile sensor Newscan systems (Nitta), to measure the distribution of plantar pressure. Because the F-SCAN uses an extremely thin sensor $(0.15 \mathrm{~mm})$, more accurate measurments may be obtained. This sensor was attached to the sole of shoes, and plantar pressure in these subjects was measured by having them wear shoes with these sensor sheets coordinated to the size of shoes in the respective individuals. The tip of the sensor sheet was easily connected to a portable sensor unit fixed at the leg using a cuff band. Analogue signals from sensors were transferred to a computer via a sensor unit, and converted to digital signals. Calibration was performed by having subjects stand on one leg, the load per unit area $\left(\mathrm{kg} / \mathrm{cm}^{2}\right)$ was corrected. All subjects walked for $7 \mathrm{~m}$ on a hard flat floor five times at their normal walking speed, and then load pressure was measured. Walking speed such as cadence was not established so as not to impair gait style of each subject. Among load pressures over 4 seconds of natural walking, the third and fourth steps were selected for analysis to determine the mean stance phase during one walking cycle, as the walking speed is stable during this period (Fig. 1).

The types of central loci of the plantar pressure were analyzed in the two groups, and the total weight-bearing between heel contact and toe off was measured by evaluating the stance phase during one walking cycle in the respective types of loci. In addition, the plantar region was divided into the following 4 compartments (at a ratio of $3: 3: 2: 2$ ): heel, plantar arch, anterior foot, and toe. Then changes in load pressure (integrate: $\mathrm{kg} / \mathrm{cm}^{2} \cdot \mathrm{s}$ ) and load time (seconds) were measured at the respective compartments (Fig. 2, 3). Integrates at the respective 4 compartments were compared after correcting integrates for weight differences in the respective individuals. That is, the total integrate at 4 compartments was considered to be $100 \%$, and the rate (\%) of integrates at the respective compartments was calculated (Fig. 4). For the load time, the interval between heel contact and toe off was considered to be $100 \%$, and the rate (\%) of load time at the respective compartments to the former interval was calculated.

\section{Measurement of kyphosis}




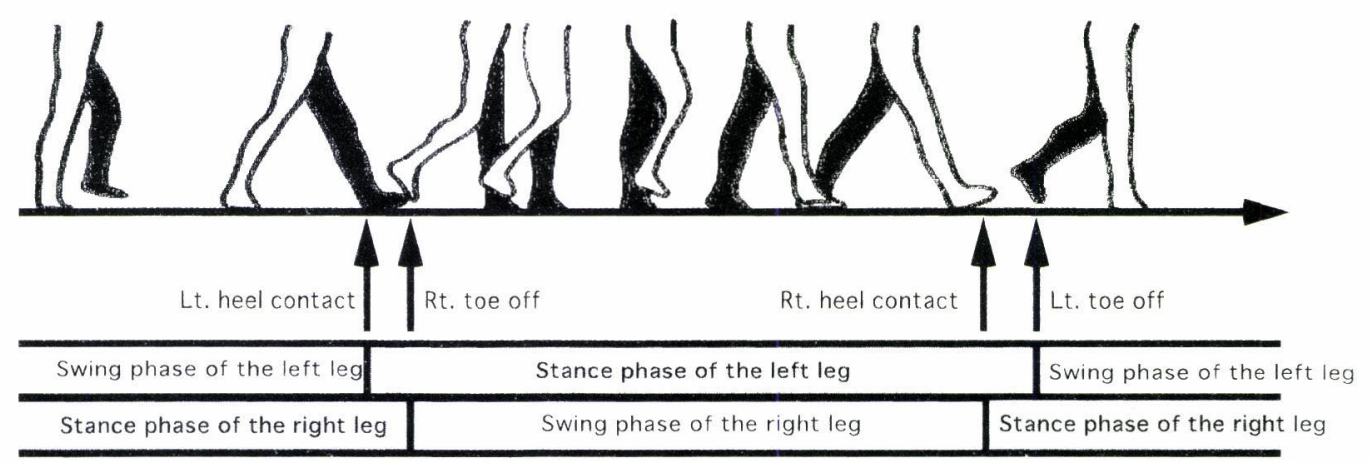

Lt. single support

double support

Fig. 1 One average gait cycle.

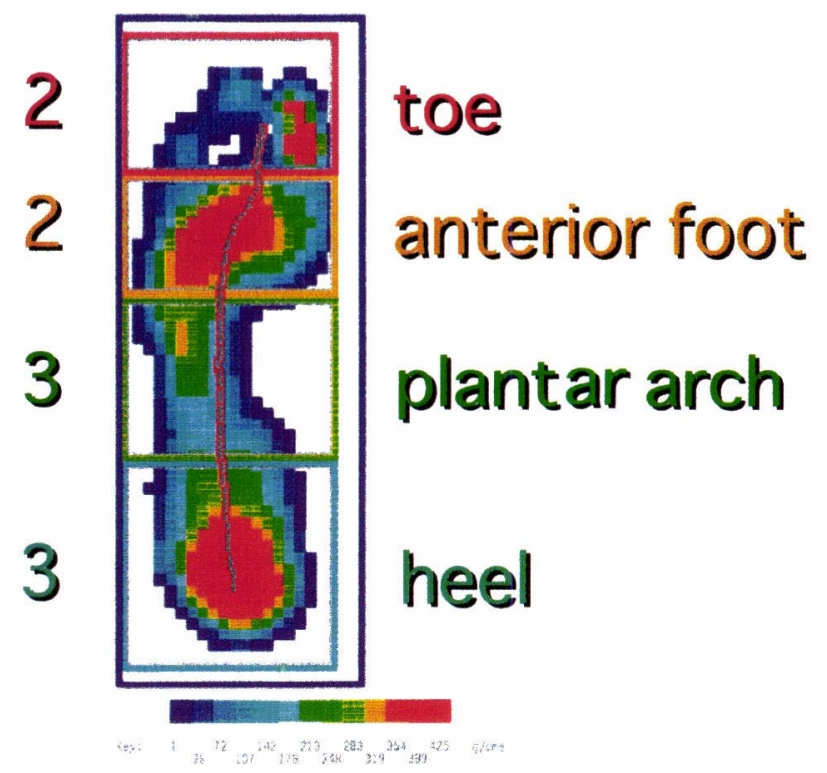

Fig. 2 Distribution of load pressure in a healthy subject (left leg).

The central red zone represents the central locus of the plantar pressure.

The subjects grasped a supporting bar by elevating the upper limbs at 30 degrees in the forward direction to assume a natural standing position parallel to the orbito-auricular line. Using a large X-ray film, lateral roentgenograms of the total spine from the ear to the sacral bone were obtained in the standing position for structural evaluation of the resting spinal column ${ }^{1,2)}$. That is, plumb-line shift from the central sacral line (P-CSL: $\mathrm{mm}$ ) was used in the analysis (Fig. 5).

Based on these values, we evaluated whether structural changes in the spinal column were correlated with the central locus of the plantar pressure or integrates and load times at the 


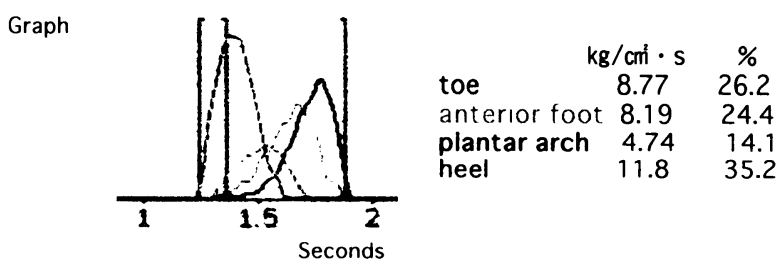

Integrates of load pressure at 4 compartments during the stance phase of one selected walking cycle (left leg) are shown on the right side of this graph. Integrates at 4 compartments (respectively expressed by 4 different colors) are equivalent to areas in this graph. [the ordinate represents load pressure $(\mathrm{kg} / \mathrm{cm} 2)$ and the abscissa represents the time (seconds). Three walking cycles can be observed in approximately 4 seconds]

Fig. 3 Dynamic changes and integrates of load pressure.

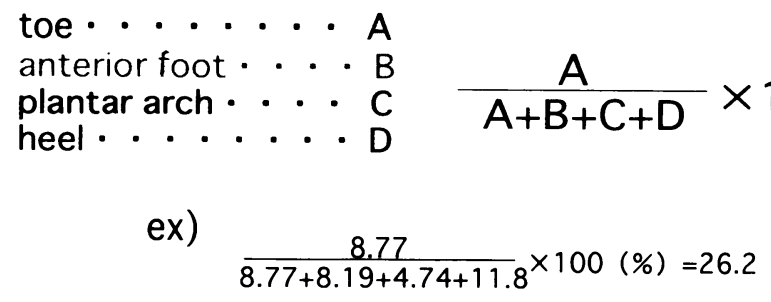

$$
\text { ex) } \frac{8.77}{8.77+8.19+4.74+11.8} \times 100(\%)=26.2
$$

Weight differences in the respective subjects were corrected to evaluate integrates at 4 compartments. The total integrate of load pressure at 4 compartments was considered to be $100 \%$, and the rate $(\%)$ of load pressure at the respective compartments was calculated. For example, to calculate the rate $(\%)$ of load pressured at the toe (red compartment), integrate at compartment $A(\mathrm{~kg} / \mathrm{cm} 2)$ was divided by the total integrate at 4 compartments $(A+B+C+D)$. As shown in this figure, the rate of load pressure at the toe (red compartment) was $26.2 \%$.

Fig. 4 Method of calculating the rate of weight-bearing.

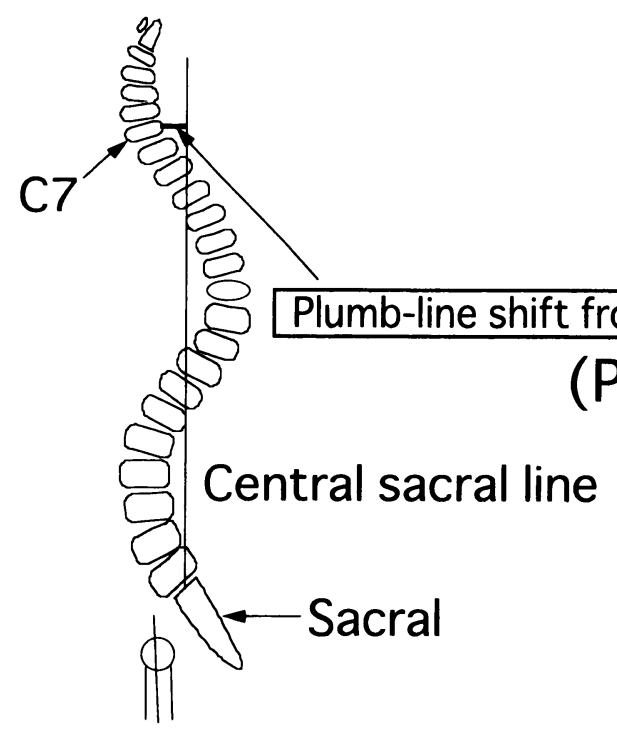

Fig. 5 Plumb-line shift from the central sacral line. 


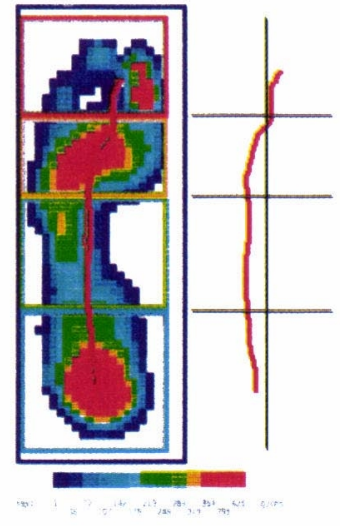

Control $(n=20)$

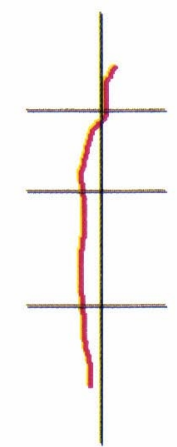

Normal type

$19(52.8 \%)$

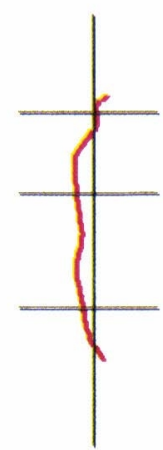

Short and unstable type 9(25.0\%)

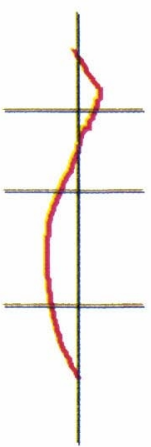

Lateral convex type $8(22.2 \%)$

Kyphotic group $(\mathrm{n}=36)$

Fig. 6 Comparison of the central locus of the plantar pressure between control and kyphotic patients.

respective compartments when the central locus of the plantar pressure was classified into the respective types of kyphosis. All values were tested by Student's t-test, and a $\mathbf{P}$ valve of less than $5 \%$ was considered significant.

\section{Results}

\section{The central locus of plantar pressure}

\section{1) Control}

When the central locus of plantar pressure was evaluated, the center of pressure started to move from the center of the heel, and the period of heel contact was prolonged. Subsequently, the center of pressure smoothly shifted in the forward direction by outwardly drawing a slightly convex arch. The center of pressure demonstrated a slightly prolonged period during toe off, but was not fixed to the same location as observed during heel contact. Then, the center of pressure slowly shifted toward the hallux (Fig. 6). In the control group, all subjects showed identical patterns in the central locus of plantar pressure.

2) Kyphotic patients

When the central locus of plantar pressure was classified into three types based on the pattern of changes during walking types, the extent of changes correlated with the severity of kyphosis.

(1) Normal type

Patients with normal type kyphosis (19 patients : 52.8\%) showed a similar central locus of the plantar pressure to the control.

(2) Short and unstable type

Times corresponding to both heel contact and toe off were short, and the central locus of the plantar pressure was short in patients with short and unstable type kyphosis ( 9 patients : $25.0 \%$ ). Moreover, left and right amplitudes were small and unstable.

(3) Lateral convex type 
Table 1 Comparison of the integrates $(\%)$ at the respective plantar compartments between the control and kyphotic groups.

\begin{tabular}{|c|c|c|c|c|}
\hline & \multirow{2}{*}{$\begin{array}{l}\text { Control } \\
(n=20)\end{array}$} & \multicolumn{3}{|c|}{$\begin{array}{l}\text { Kyphotic group } \\
\qquad(\mathrm{n}=36)\end{array}$} \\
\hline & & $\begin{array}{l}\text { Normal type } \\
\quad(n=19)\end{array}$ & $\begin{array}{l}\text { Short and unstable type } \\
\qquad(n=9)\end{array}$ & $\begin{array}{l}\text { Lateral convex type } \\
\qquad(\mathrm{n}=8)\end{array}$ \\
\hline heel $(\%)$ & $37.2 \pm 2.7$ & $36.2 \pm 2.8 \mathrm{NS}$ & $35.2 \pm 1.8 \mathrm{NS}$ & $40.6 \pm 2.8^{*}$ \\
\hline plantar arch (\%) & $17.4 \pm 2.4$ & $18.7 \pm 2.5 \mathrm{NS}$ & $19.0 \pm 1.5 \mathrm{NS}$ & $19.7 \pm 2.5 \mathrm{NS}$ \\
\hline anterior foot $(\%)$ & $33.5 \pm 4.1$ & $32.0 \pm 4.2 \mathrm{NS}$ & $33.1 \pm 4.2 \mathrm{NS}$ & $31.1 \pm 4.2 \mathrm{NS}$ \\
\hline toe $(\%)$ & $11.9 \pm 2.9$ & $13.1 \pm 3.0 \mathrm{NS}$ & $12.7 \pm 3.0 \mathrm{NS}$ & $8.6 \pm 3.0 \mathrm{NS}$ \\
\hline
\end{tabular}

(NS : not significant, ${ }^{*}: \mathrm{p}<0.05$ )

The time corresponding to heel contact was longer in patients with lateral convex type kyphosis ( 8 patients : $22.2 \%$ ) than in the control. The central locus of the plantar pressure in these patients made a slight outward convex arch in the forward direction, and then made an inward convex arch. However, the terminal point of the central locus was shifted toward the outside around the anterior foot.

\section{Integrates at the respective plantar compartments}

\section{1) Control}

The mean rate $(\%)$ of weight-bearing at each of the 4 plantar compartments was : $37.2 \pm$ $2.7 \%$ at the heel, $17.4 \pm 2.4 \%$ at the plantar arch, $33.5 \pm 4.1 \%$ at the anterior foot, and $11.9 \pm 2.9 \%$ at the toe (Table 1 ).

\section{2) Kyphotic patients}

Integrates of load pressure were compared between patients with the 3 different types of kyphosis.

(1) Normal type

The mean weight-bearing rates were : $36.2 \pm 2.8 \%$ at the heel, $18.7 \pm 2.5 \%$ at the plantar arch, $32.0 \pm 4.2 \%$ at the anterior foot, and $13.1 \pm 3.0 \%$ at the toe. There were no significant differences in the rate of weight-bearing at any compartment between patients with normal type kyphosis and controls (Fig. 7).

(2) Short and unstable type

The mean weight-bearing rates were $35.2 \pm 1.8 \%$ at the heel, $19.0 \pm 1.5 \%$ at the plantar arch, $33.1 \pm 4.2 \%$ at the anterior foot, and $12.7 \pm 3.0 \%$ at the toe. These were not significantly different from control values (Fig. 8).

(3) Lateral convex type

The mean weight-bearing rates were $40.6 \pm 2.8 \%$ at the heel, $19.7 \pm 2.5 \%$ at the plantar arch, $31.1 \pm 4.2 \%$ at the anterior foot, and $8.6 \pm 3.0 \%$ at the toe. The rate of weightbearing at the heel was significantly larger in patients with lateral convex type kyphosis than in the controls $(\mathrm{p}<0.05)$ (Fig. 9)

\section{Load times at the respective compartments}

In the present study, central changes in load pressure over 0.02 -second intervals between heel contact and toe off were expressed as the locus of plantar pressure to measure the load 


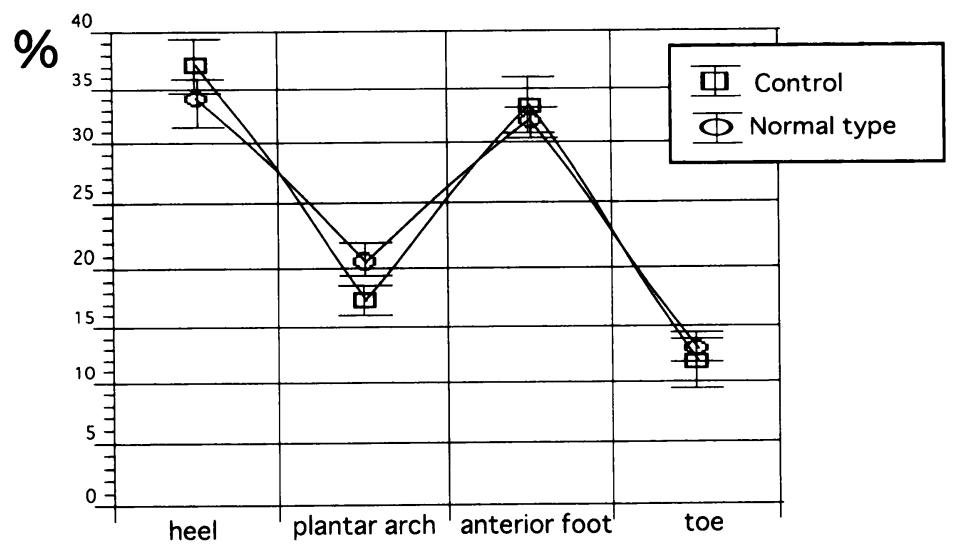

Fig. 7 Comparison of the integrates $(\%)$ at the respective plantar compartments between the control and normal type.

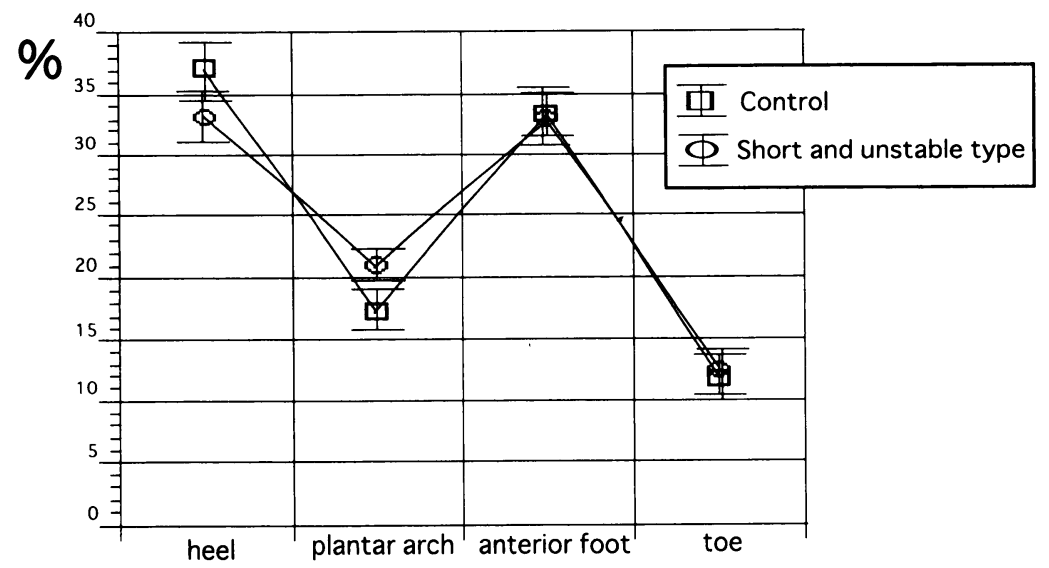

Fig. 8 Comparison of the integrates $(\%)$ at the respective plantar compartments between the control and short and unstable type.

time. Because the walking cycle differed from individual to individual, the rate of load time at the respective compartments was calculated.

1) Control

The mean load time rate was $30.4 \pm 3.7 \%$ at the heel, $23.5 \pm 4.2 \%$ at the plantar arch, $34.3 \pm 4.1 \%$ at the anterior foot, and $11.8 \pm 3.9 \%$ at the toe (Table 2).

2) Kyphotic patients

(1) Normal type

The mean load time rate was $32.3 \pm 2.8 \%$ at the heel, $21.7 \pm 2.5 \%$ at the plantar arch, $32.1 \pm 3.2 \%$ at the anterior foot, and $13.9 \pm 3.0 \%$ at the toe. None of these valves were different from control valves (Fig. 10).

(2) Short and unstable type

The mean load time rate was $32.6 \pm 5.8 \%$ at the heel, $25.7 \pm 4.5 \%$ at the plantar arch, $28.1 \pm 4.6 \%$ at the anterior foot, and $13.6 \pm 4.0 \%$ at the toe. The load time rate at the anterior foot was significantly smaller in patients with short and unstalbe type kyphosis than 


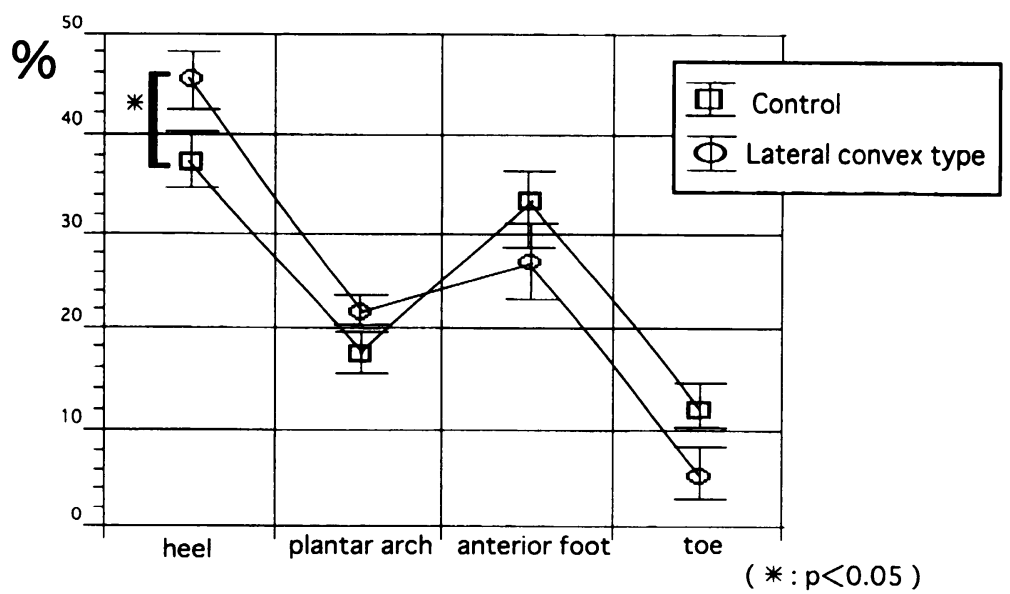

Fig. 9 Comparison of the integrates (\%) at the respective plantar compartments between the control and lateral convex type.

Table 2 Comparison of load times $(\%)$ at the respective compartments between the control and kyphotic groups.

\begin{tabular}{|c|c|c|c|c|}
\hline & \multirow{2}{*}{$\begin{array}{l}\text { Control } \\
(n=20)\end{array}$} & \multicolumn{3}{|c|}{$\begin{array}{l}\text { Kyphotic group } \\
\quad(n=36)\end{array}$} \\
\hline & & $\begin{array}{l}\text { Normal type } \\
\quad(n=19)\end{array}$ & $\begin{array}{l}\text { Short and unstable type } \\
\qquad(n=9)\end{array}$ & $\begin{array}{l}\text { Lateral convex type } \\
\qquad(\mathrm{n}=8)\end{array}$ \\
\hline heel $(\%)$ & $30.4 \pm 3.7$ & $32.3 \pm 2.8 \mathrm{NS}$ & $32.6 \pm 5.8 \mathrm{NS}$ & $37.7 \pm 5.5^{*}$ \\
\hline plantar arch (\%) & $23.5 \pm 4.2$ & $21.7 \pm 2.5 \mathrm{NS}$ & $25.7 \pm 4.5 \mathrm{NS}$ & $20.7 \pm 5.5 \mathrm{NS}$ \\
\hline anterior foot $(\%)$ & $34.3 \pm 4.1$ & $32.1 \pm 3.2 \mathrm{NS}$ & $28.1 \pm 4.6 \mathrm{NS}$ & $31.1 \pm 6.2 \mathrm{NS}$ \\
\hline toe $(\%)$ & $11.8 \pm 3.9$ & $13.9 \pm 3.0 \mathrm{NS}$ & $13.6 \pm 4.0 \mathrm{NS}$ & $10.5 \pm 3.9 \mathrm{NS}$ \\
\hline
\end{tabular}

(NS : not significant, ${ }^{*}: \mathrm{p}<0.05$ )

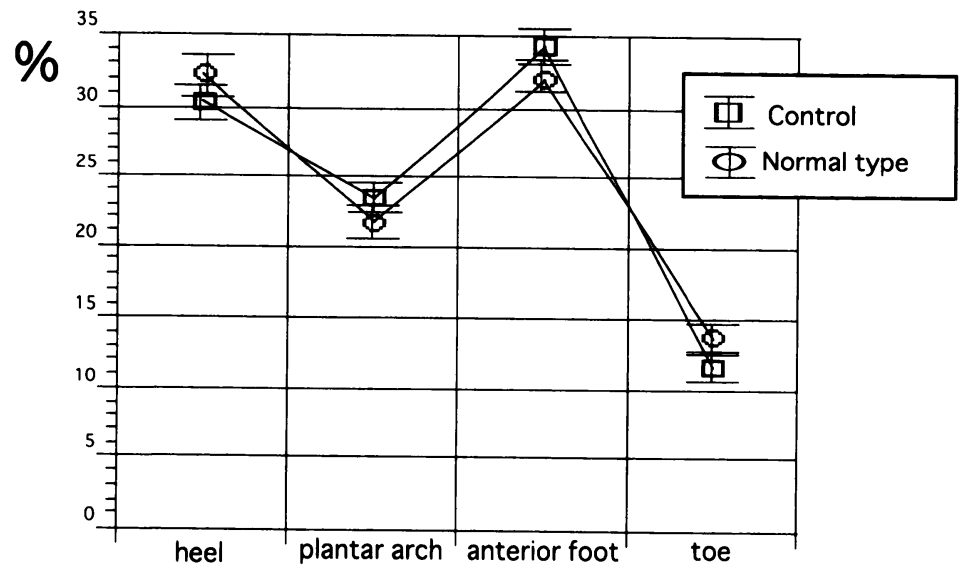

Fig. 10 Comparison of load times $(\%)$ at the respective compartments between the control and normal type. 


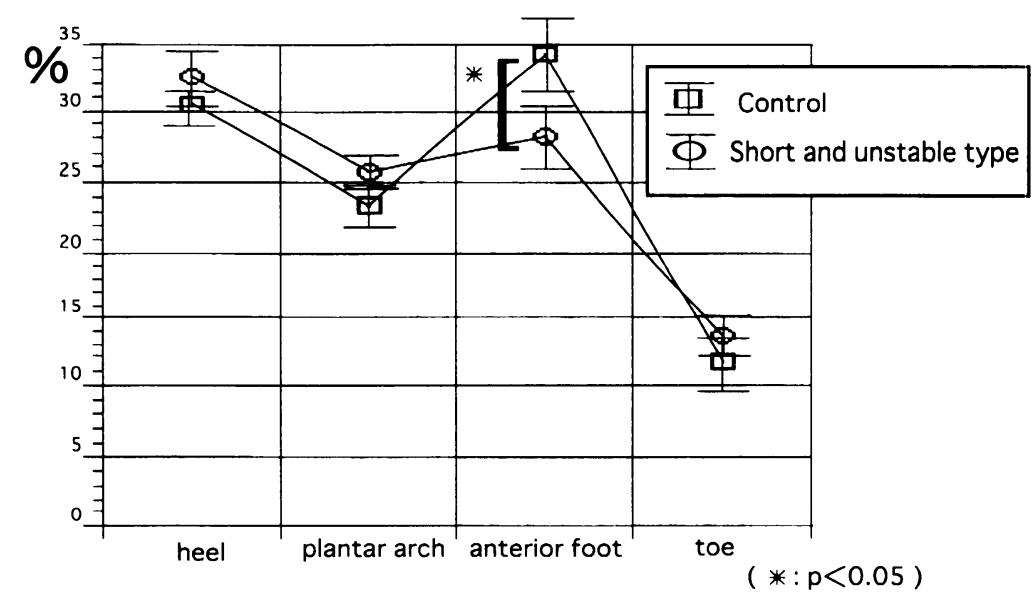

Fig. 11 Comparison of load times $(\%)$ at the respective compartments between the control and short and unstable type.

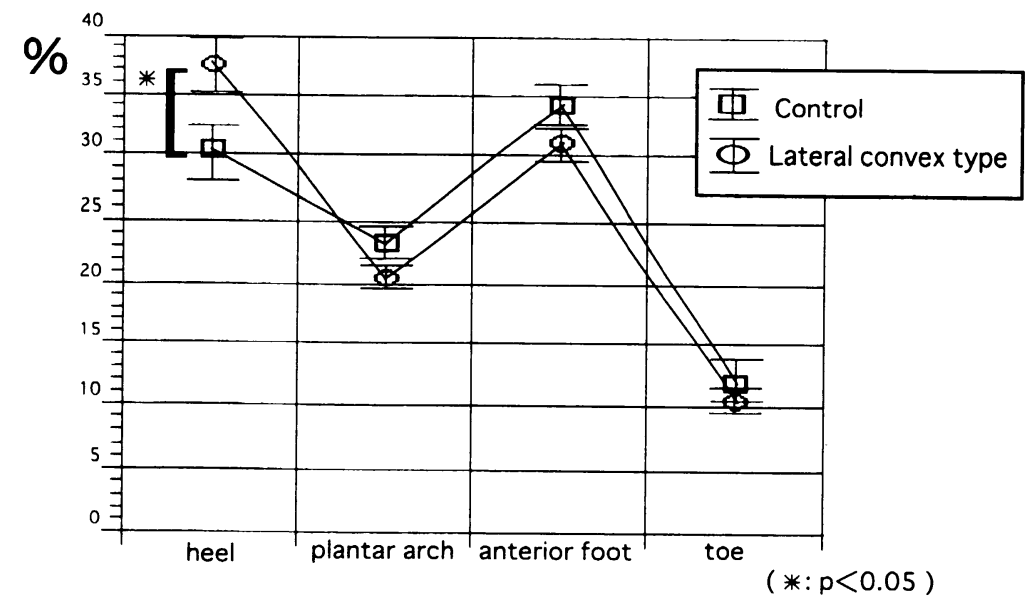

Fig. 12 Comparison of load times $(\%)$ at the respective compartments between the control and lateral convex type.

in the controls $(\mathrm{p}<0.05)$ (Fig. 11).

(3) Lateral convex type

The mean load time rate was $37.7 \pm 5.5 \%$ at the heel, $20.7 \pm 5.5 \%$ at the plantar arch, $31.1 \pm 6.2 \%$ at the anterior foot, and $10.5 \pm 3.9 \%$ at the toe. The rate of load time at the heel was significantly larger in patients with lateral convex type kyphosis than in the controls $(\mathrm{p}<0.05)$ (Fig. 12).

\section{Structural changes in the spinal column}

The mean value for P-CSL was $1.1 \pm 5.7 \mathrm{~mm}$ in the controls, $1.0 \pm 6.8 \mathrm{~mm}$ in patients with normal type kyphosis, $3.3 \pm 8.8 \mathrm{~mm}$ in patients with short and unstable type kyphosis, and $13.8 \pm 8.1 \mathrm{~mm}$ in patients with lateral convex type kyphosis. Patients with lateral convex type kyphosis had significantly larger anterior P-CSL than controls (Table 3).

The P-CSL and load time at the heel were not significantly correlated in patients with 
Table 3 Comparison of P-CSL between the control and the kyphotic groups.

\begin{tabular}{|c|c|c|c|c|}
\hline & \multirow{2}{*}{$\begin{array}{l}\text { Control } \\
(n=20)\end{array}$} & \multicolumn{3}{|c|}{$\begin{array}{l}\text { Kyphotic group } \\
\quad(n=36)\end{array}$} \\
\hline & & $\begin{array}{l}\text { Normal type } \\
\quad(n=19)\end{array}$ & $\begin{array}{l}\text { Short and unstable type } \\
\qquad(n=9)\end{array}$ & $\begin{array}{l}\text { Lateral convex type } \\
\qquad(n=8)\end{array}$ \\
\hline P-CSL $(\mathrm{mm})$ & $1.1 \pm 5.7$ & $1.0 \pm 6.8 \mathrm{NS}$ & $3.3 \pm 8.8 \mathrm{NS}$ & $13.8 \pm 8.1^{*}$ \\
\hline
\end{tabular}

P-CSL : Plumb-line shift from the central sacral line

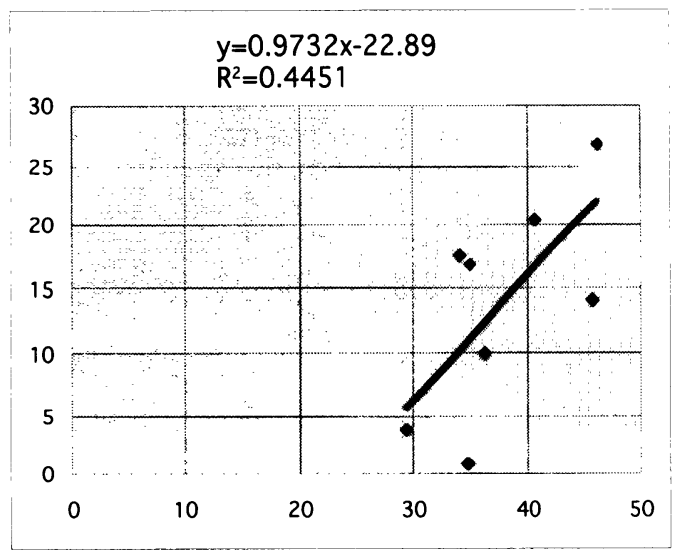

Fig. 13 Correlation between P-CSL and load time at the heel was evaluated in patients with lateral convex type kyphosis.

lateral convex type kyphosis, but there was a positive correlation between the two $(\mathrm{p}<0.1)$ (Fig. 13).

\section{Discussion}

\section{Characteristics of the F-SCAN system}

In the present study we analyzed walking using an F-SCAN ${ }^{3-5)}$. Compared to a conventional force plate, the small and lightweight F-SCAN did not affect gait in any subject. Therefore, the F-SCAN facilitated measurement of load pressure during more natural walking. Moreover, the F-SCAN sensor sheets contain circuits equipped with flexible electrodes, and can be freely cut according to the size of the individual foot. Sensor sheets for the F-SCAN can also be attached to the insole of shoes. The distribution of plantar pressure during standing and walking, and peak values for regional high-pressure regions can be instantly measured by the F-SCAN. Therefore, the F-SCAN is well-suited for pre- and postoperative evaluations and postoperative treatment of hallux valgus and gonarthrosis $^{6}$. Moreover, the F-SCAN can detect changes in walking environment such as steps and slopes, as well as providing real-time observation of images. Results obtained with the F-SCAN are expressed by different colors and are therefore easily understood by patients. However, sensor sheets for the F-SCAN have the following disadvantages : sensor 
sheets are not durable; reaction of resistant ink does not corrrespond to changes in load pressure; and plantar temperature or distortion of sensor sheets caused by extended measurement easily induce errors during measurement ${ }^{7}$.

\section{Comparison with other measurement procedures}

At present, methods of measuring locomotion are roughly classified into kinematic and kinetic procedures. Previous kinematic procedures included photographic procedures, cinematographic procedures, and vasogram. In recent years, procedures such as Polgon and telemetric real-time acquisition of kinematic system (TRACK) have been developed for kinematic evaluations with developments in computing ${ }^{8}$

Kinetic procedures include a force plate and devices for measuring the distribution of plantar pressure. A force plate facilitates recording and storage of objective data of walking, and these data can be compared to those obtained at other facilities. Moreover, a force plate also facilitates understanding of abnormality and changes that cannot be subjectively and grossly evaluated by examiners ${ }^{9}$.

\section{Comparison between F-SCAN and force plate}

1) Structures and measurable component forces of load pressure

A force plate basically consists of an aluminum top plate and base frame between which isosensitive quartz transducers are located at 4 corners to measure three component forces. Therefore, vertical, anteroposterior, and lateral forces can be measured, as well as various other parameters such as impulse, speed and displacement of the centroid, and COP. On the other hand, sensor sheets for the F-SCAN are made of polyester film coated with a thin membrane of pressure resistant material, and the matrix of electrodes are distributed at regular intervals on both upper and lower sides of polyester film. Therefore, when the sensor sheet comes into contact with the subject, load pressure is periodically scanned at the respective intersections to measure the distribution of plantar pressure (Fig. 14). Although the F-SCAN is structurally easy to use, anteroposterior and lateral forces cannot be measured by these sensor sheets. However, vertical force can be measured by the F-SCAN when the system is correctly calibrated. Subsequently, vertical force can be variously expressed by impulse or COP (Fig. 15).

The vertical force of load pressure is a reaction force against the resultant force of gravity and inertial force induced by upward and downward movements of the body. Because both acceleration of gravity and body mass are constant during walking, the vertical force of load pressure changes in proportion to vertical accelerative components of inertial force according to the level of body weight. Anteroposterior force of load pressure is closely correlated with stride, while lateral force changes with the magnitude of stride ${ }^{10)}$. When reproducible walking cannot be expected because of dysbasia, data from such patients cannot be compared. Because vertical force is not markedly influenced by differences in gait, and values for vertical force are larger than those of the other 2 component forces, minute changes in vertical force are evaluable. Therefore, the F-SCAN is easier and superior to a force plate, because the vertical force of load pressure can be measured. However, the F-SCAN is not suitable for comparison of absolute values, but is useful for analyzing relative values such as a distribution pattern of pressure. Therefore, we selected the F-SCAN to analyze load pressure in the present study ${ }^{11}$. 

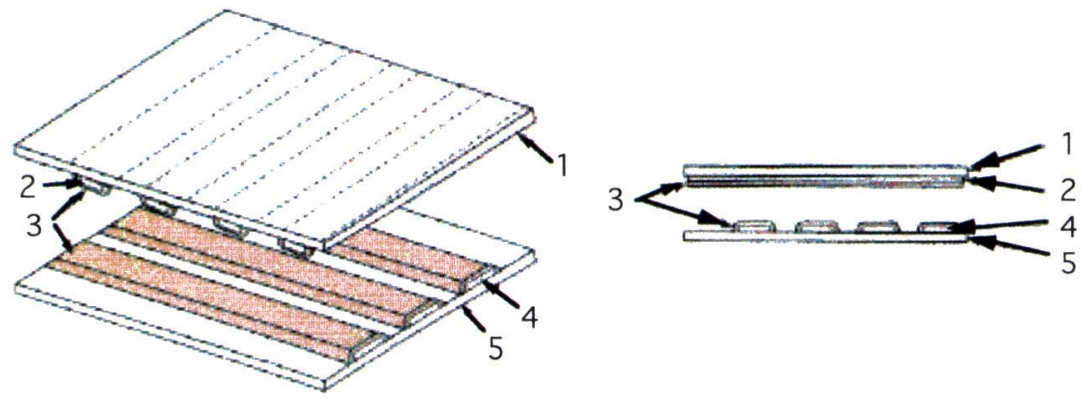

1: Base sheet at the side of line electrodes (polyester film)

2: Line electrodes

3: Special ink

4: Row electrodes

5: Base sheet at the side of row electrodes (polyester film)

Fig. 14 Construction of sensor sheet (taken from reference 3 )
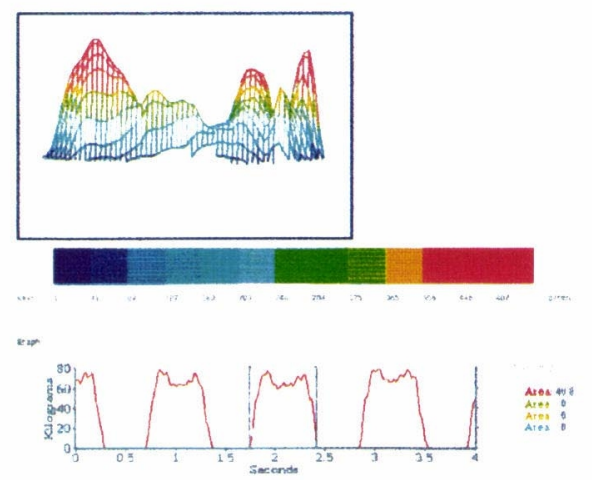

Graph of the entire planta pedis in one leg. Three-dimensional expression of both legs.
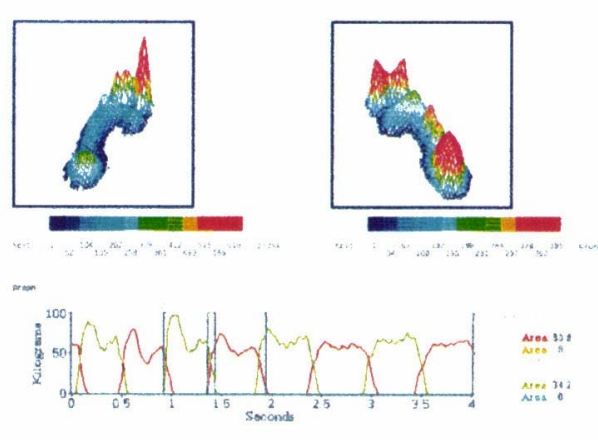

Three-dimensional expression of both legs.

Fig. 15 Graphic reconstruction of acquired vertical force

\section{2) Evaluation of load pressure is not influenced by cadence}

Parameters of gait such as cadence need not be established when usual and natural gait style is evaluated in kyphotic patients, because these tests may alter the natural gait of the kyphotic patient. Using the F-SCAN, the rate of weight-bearing was evaluated without establishing cadence. That is, the rate of weight-bearing was calculated from integrates, not from peak values. When peak values are used to calculate the rate of load pressure after establishment of cadence, bimodal peaks in analogue waveforms of vertical force increase with the magnitude of cadence, while a trough between the two peaks decreases with the magnitude of cadence. In contrast, bimodal peaks decreases with a reduction of cadence, while the trough between the two peaks greatly increases with the reduction of cadence $\left(\right.$ Fig. 16) ${ }^{12)}$.

Integrates, that is impulses (changes in momentum), can be obtained by integrating the respective component forces by time, which actually correspond to the area in analogue 

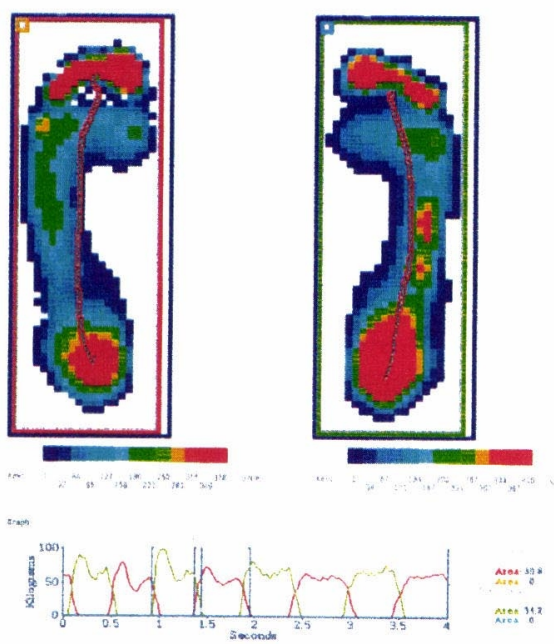

This graph shows analogue waveforms obtained by gradually decreasing cadence in a healthy subject over 4 seconds. Bimodal peaks of load pressure (vertical forces) in both legs were respectively altered to trapezoidal forms.

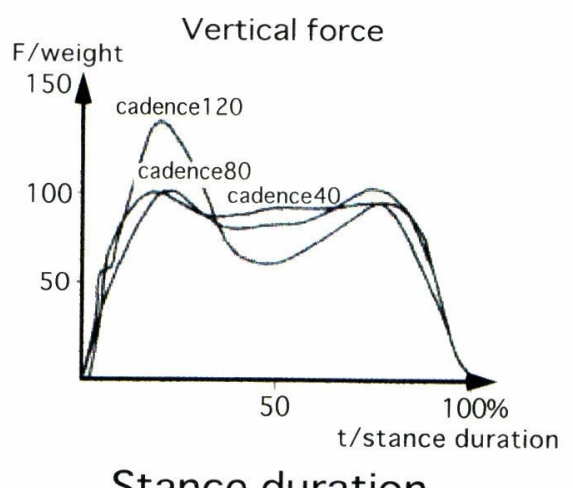

Cadence-dependent waveforms of vertical force obtained by a force plate. As observed by the F-SCAN, the graph of vertical force was altered to a trapezoidal form with the decrease in cadence.

Fig. 16 Changes of vertical force with cadence.

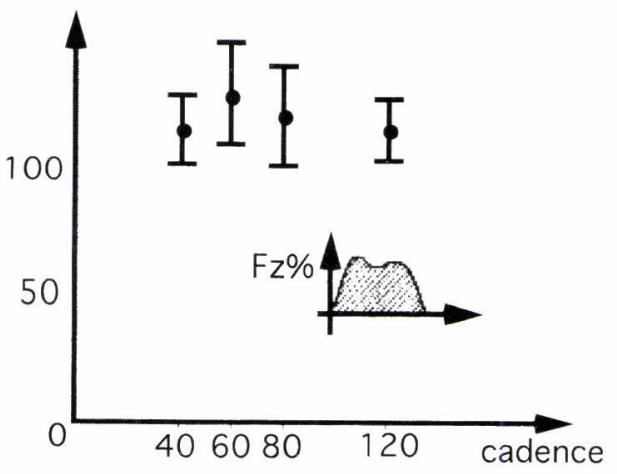

Integrates (impulses) representing changes in vertical force did not change with cadence.

Fig. 17 Integrated vertical force (taken from reference 8).

waveforms obtained by measuring floor reaction force. However, the vertical component of integrates is not markedly affected by cadence (Fig. 17) ${ }^{8}$. That is, when kyphotic patients with various gait styles are studied, the use of a cadence-independent integrate facilitates more accurate analysis when the rate of weight-bearing is determined by vertical force obtained by the F-SCAN. However, the vertical force of load pressure also decreases during walking with a slower cadence of 40 steps per minute (cadence 40), and then lateral swaying increases, easily inducing errors. Therefore, all patients were forced to walk naturally as far as possible during examination in the present study. 


\section{Characteristics of walking in kyphotic patients}

\section{1) Lateral swaying}

As described above, it is difficult to analyze lateral components of load pressure with the F-SCAN. However, when the locus is used, certain stability can be estimated. In the present study, the central locus of plantar pressure, which can be used as a standard of lateral stability, did not change significantly in kyphotic patients. Even in the control, however, lateral components of waveforms of floor reaction exhibit sharp peaks representing an impulse induced by heel contact, and subsequently exhibit gently-sloping waveforms, which result in swaying. Values for such lateral components are smaller than those for vertical and anteroposterior components. Therefore, lateral components were not evaluated sufficiently because of various problems including errors, individual differences, differences between the left and right, and reproducibility ${ }^{8)}$. Therefore, it is difficult to precisely analyze the central locus of plantar pressure with the F-SCAN, even when swaying or lateral stability of walking can be evaluated by the F-SCAN. However, lateral shifting (amplitude) of the hip joint increased and resulted in swaying gait, which was considered to be reflected to the central locus of the plantar pressure when the pelvis is shifted to the axis of the supporting leg in elderly patients with lateral convex-type kyphosis demonstrating outwardly convex arch of the locus. Such kyphotic patients were more frequently complicated by limitation and contracture of hip joint excursion than controls.

\section{2) Anteroposterior balance of body gravity}

In the present study, 4 compartments were established in the planta pedis in the anteroposterior direction, and load times were measured to evaluate anteroposterior balance during walking. The load time at the anterior foot was shortened during the driving phase in patients with short and unstable type kyphosis, demonstrating a decreased central locus of plantar pressure. Subsequently, the shortened load time accelerates heel contact of the contralateral leg, and prolongs load times in both legs, resulting in stable walking. Even in patients with lateral convex-type kyphosis, load times at the heel were prolonged, also resulting in stable walking.

3) Correspondence with structural changes in the spinal column

When patients show a gait style that markedly differed from that in normal subjects, walking ability in such patients is generally evaluated as poor ${ }^{9}$. At present, there is no absolute standard for evaluation of walking in kyphotic patients. However, walking ability in kyphotic patients generally decreases with the severity of spinal deformity. Therefore, the present study used P-CSL as an index for evaluating the severity of the deformity. P-CSL was correlated with changes in load pressure. Kyphotic patients maintain their balance in the anteroposterior directions by posterior shifting of gravity, that is anterior P-CSL. Therefore, the rate of weight-bearing at the heel obtained by the F-SCAN was increased in kyphotic patients. During natural standing, the point of application of body gravity at the planta pedis is identical between normal subjects and kyphotic patients. Thus, it was considered that kyphotic patients maintain their balance in the anteroposterior directions by prolongation of load times, that is increased integrates at the heel. The interval between heel contact and plantar contact increases with the magnitude of anterior P-CSL. The interval between heel contact and plantar contact tended to increase with the degree of anterior P-CSL. 


\section{Future applications of the F-SCAN system}

Although walking has long been analyzed using a force, this procedure is not yet sufficiently accepted in clinical settings, because the force plate itself was not previously sufficiently precise, and data obtained using a force plate could not be compared with those obtained at other facilities ${ }^{9)}$. However, the F-SCAN is easy to use and facilitates subjective and functional evaluation by conventional roentgenography, which provides an objective index for evaluating therapeutic values. Because data obtained by the F-SCAN can be variously expressed, the F-SCAN is expected to be widely used in the future for the development of sports shoes or rehabilitation of patients with gait disturbance caused by hemiplegia.

\section{Acknowledgments}

A part of this study was presented at the 71st scientific meeting of the Japanese Orthopaedic Association and the 21 st World Triennial Congress of Societe Internationale de Chirurgie Orthopedique et de Traumatologie (SICOT).

\section{References}

1) Dickson RA, Lawton JO, Archer IA and Butt WP: The pathogenesis of idiopathic scoliosis. J. Bone Joint Surg, $66: 8-15$ (1984)

2) Inoue $\mathrm{K}$ : The sagittal curvature of spine in idiopathic scoliosis. - Its morphological features and the correlation among sagittal and frontal curvature and rotation of apical vertebra-. Nippon Seikeigeka Gakkai zasshi, 59 : 505-516(1985)(In Japanese)

3) Azuma T: The new foot pressure analyzing system F-SCAN. PO Academy Journal, 2 : 167-171(1994) (In Japanese)

4) Makikawa K, Takenaka Z, Ashihara $S$ and Nanagawa $K$ : Dynamic pressure distribution under the foot in various modes of walking. Seikeigeka Biomechanics, 5 : 249-252(1983) (In Japanese)

5) Ando N, Ando M, Mano Y, Suzumura A, Takayanagi $T$ and Takeuchi $T$ : New corrective shoes for patients with cerebellar ataxia. Rihabilitation Igaku, $35: 100-105$ (1998) (In Japanese)

6) Takuma S, Hashimoto K, Nakajima $S$ and Sawamura S : Analysis of plantar pressures under normal feet using Foot-SCAN system. Nippon Rinsyhou Biomechanics Gakkaishi, 16 : 495-500(1995) (In Japanese)

7) Ando N, Fujimoto $Y$, Ando M, Mano $Y$ and Takayanagi $T$ : A new method of gait analysis in Duchenne muscular dystrophy. Rinsyhou Shinkeigaku, 32 : 962-967(1992) (In Japanese)

8) Sakamoto K : Biomechanical analysis of hip Joint by mathematical link model and rigid body spring model. Societe Internationale de Recherche, Orthopedigue et de Traumatologie (SIROT) abstract of 5th General Meeting, $17: 267(1990)$

9) Morita S, Furuya K, Yamamoto H and Okumura S: Analysis of hemiplegic gait with measurement of floor reaction force. Sogo Rihabilitation, $17: 771-775$ (1989) (In Japanese)

10) Morita S : Analysis of the fore-aft and lateral components of the floor reaction force in normal gait. Nippon Seikeigeka Gakkai Zasshi, 62 : 1221-1232(1988)(In Japanese)

11) Shiba N : Shock-absorbing effect of shoe insert materials commonly used in management of extremity disorders. Rinsyhou Seikeigeka, 310 : 130-136(1995)

12) Moriai N : Kinetics of human locomotion. Sogo Rihabilitation, 15 : 289-298(1987) (In Japanese)

[Received January 20, 1999 : Accepted January 26, 1999] 